\title{
Quantitative Proteomic (iTRAQ) Analysis of 1st Trimester Maternal Plasma Samples in Pregnancies at Risk for Preeclampsia
}

\author{
Varaprasad Kolla, ${ }^{1}$ Paul Jenö, ${ }^{2}$ Suzette Moes, ${ }^{2}$ Olav Lapaire, ${ }^{1}$ \\ Irene Hoesli, ${ }^{1}$ and Sinuhe Hahn ${ }^{1,3}$ \\ ${ }^{1}$ Department of Biomedicine, University Women's Hospital, 4031 Basel, Switzerland \\ ${ }^{2}$ Biozentrum, University of Basel, 4056 Basel, Switzerland \\ ${ }^{3}$ Laboratory for Prenatal Medicine, Department of Biomedicine, University Women's Hospital, Hebelstrasse 20, \\ CH 4031 Basel, Switzerland
}

Correspondence should be addressed to Sinuhe Hahn, shahn@uhbs.ch

Received 31 May 2011; Revised 26 December 2011; Accepted 28 December 2011

Academic Editor: Saulius Butenas

Copyright () 2012 Varaprasad Kolla et al. This is an open access article distributed under the Creative Commons Attribution License, which permits unrestricted use, distribution, and reproduction in any medium, provided the original work is properly cited.

A current major obstacle is that no reliable screening markers exist to detect pregnancies at risk for preeclampsia. Quantitative proteomic analysis employing isobaric labelling (iTRAQ) has been suggested to be suitable for the detection of potential plasma biomarkers, a feature we recently verified in analysis of pregnancies with Down syndrome foetuses. We have now examined whether this approach could yield biomarkers to screen pregnancies at risk for preeclampsia. In our study, we used maternal plasma samples obtained at 12 weeks of gestation, six from women who subsequently developed preeclampsia and six with uncomplicated deliveries. In our analysis, we observed elevations in 10 proteins out of 64 proteins in the preeclampsia study group when compared to the healthy control group. These proteins included clusterin, fibrinogen, fibronectin, and angiotensinogen, increased levels of which are known to be associated with preeclampsia. An elevation in the immune-modulatory molecule, galectin 3 binding protein, was also noted. Our pilot study, therefore, indicates that quantitative proteomic iTRAQ analysis could be a useful tool for the detection of new preeclampsia screening markers.

\section{Introduction}

Preeclampsia is a major health concern, as it is a leading cause of fetal and maternal mortality and morbidity, worldwide [1]. A major clinical deficit is that no reliable screening markers exist to detect at-risk pregnancies, thereby offering no opportunity for intervening therapies prior to the onset of symptoms $[2,3]$. This is especially relevant for severe cases of early onset forms of the disorder, resulting in the delivery of premature babies, who are frequently affected by intrauterine growth retardation.

It is widely accepted that the underlying aetiology of this enigmatic disorder, characterised by sudden hypertension in previously normotensive pregnant women, involves the placenta. In this context, the placentae of early onset preeclampsia are usually characterised by abnormalities in trophoblast differentiation, lack of modification of the maternal spiral arteries, and ill-defined villous structure [4].

Recent studies have indicated that the clinical symptoms, such as hypertension and proteinuria, are largely brought about by alterations in the levels of angiogenic factors produced by the placenta such as placental growth factor (PIGF), vascular endothelial growth factor (VEGF), soluble Flt-1, and endoglin $[5,6]$ Although alterations in the levels of these cytokines do precede the onset of clinical symptoms, it is not clear how effective they will be as screening markers, as these changes occur relatively late in gestation.

A considerable effort in prenatal medicine is being devoted to the development of efficacious first trimester screening tools, to detect pregnancies at risk for fetal aneuploidies, but also pregnancy-related disorders such as preeclampsia, as this would permit sufficient time for intervention. In this 
regard, quantitative changes at 11-14 weeks of pregnancy in proteins such as ADAM-12 [7], PP-13 (placental protein13) $[8,9]$, and pregnancy-associated plasma protein-A (PAPP-A) in combination with Doppler ultrasound, have been shown to posses some predictive value in detecting cases at risk for preeclampsia [10].

Since the aetiological cascade leading to the development of manifest preeclampsia is multifactorial, it is very unlikely that a single molecule (or small group of analytes) will possess sufficient sensitivity and specificity. Hence, new strategies will need to be explored in order to increase the pool of biomarker candidates.

One such route is by proteomic analysis of the maternal plasma proteome. The underling rationale for such investigations is that as the placenta is in direct contact with the maternal circulation, proteins released by it should be detectable in maternal plasma. Since preeclampsia is associated with structural placental anomalies, the underlying alterations in protein expression should be reflected in plasma proteome. Consequently, this serves as a promising route for the detection of biomarkers indicative of abnormal placentation [11].

Consequently, a recent study carried out under this premise [12] has shown that alterations in protein expression are evident maternal serum or plasma of affected pregnancies. Unfortunately the validity of these studies is limited, as they were performed on samples obtained from cases with manifest symptoms and, furthermore, did not employ state-of-the-art quantitative approaches permitting precise assessment of the extent of up- or downregulation of the newly discovered markers.

A further limitation in these studies is the extremely complex nature of the plasma/serum proteome, whereby low-abundance proteins are masked by the preponderance of a small number of highly abundant plasma proteins. In addition, the high dynamic range of the plasma proteome precludes the use of conventional proteomic strategies employing gel electrophoresis, even when making use of fluorescent labeling, for example, DIGE (difference gel electrophoresis). Hence, the identification of new biomarkers in plasma/serum by conventional proteomic approaches is not very feasible.

A method that has been proposed to largely overcome these deficits is isobaric labelling (iTRAQ: isobaric tags for absolute and relative quantitation) coupled with MALDITOF/TOF analysis. In this approach, the plasma peptides, following trypsin digestion, are chemically labelled with a discrete set of isotopes via an amine-tagging reagent [13]. These isotope tags permit ready discrimination by mass spectrometry, thereby permitting comparative quantification to a reference sample in a multiplex manner. To date, most applications have made use of commercial 4-plex or 8plex reagents. By the use of these different isobaric tags, it is possible to examine different samples in a single mass spectrometric analysis.

Since this method has been shown to permit highly reproducible comparative assessment of tagged peptides [14], it has been suggested to be suitable for the discovery of biomarkers in complex body fluid such as plasma [15].
In a recent study we have shown that the iTRAQ isobaric labelling approach may indeed be valid for the detection of biomarkers for pregnancy related conditions, such as fetuses with Down syndrome, in that we were able to discern quantitative alterations in known screening markers such as $\beta$ hCG [16].

Confident by the validity of the iTRAQ methodology, we have now in a pilot experiment investigated whether this approach could be suitable for the detection of biomarkers useful for determining pregnancies at risk for preeclampsia. In this study we examined 12 maternal plasma samples obtained at 12 weeks of pregnancy, of which 6 cases subsequently developed preeclampsia, while 6 cases had uncomplicated deliveries.

Maternal plasma peptides were examined by 4 -plex iTRAQ labelling in conjunction with a 4800 MALDITOF/TOF analysis. Our results indicate that quantitative differences can be being already detected in the first trimester of pregnancy between pregnancies which subsequently developed preeclampsia and those which had normal healthy outcome.

\section{Materials and Methods}

2.1. Samples. Blood samples for this case-control proteome study were collected prospectively from pregnant women at approximately 12 weeks of gestation. In a retrospective manner, 6 samples from cases that subsequently developed preeclampsia were matched with 6 samples from pregnancies with normal healthy outcome (Table 1). This study was undertaken with the approval of the Institutional Ethical Board of the University Hospital, Basel, Switzerland and written informed consent was required in all instances.

2.2. Sample Preparation. As described previously, $9 \mathrm{~mL}$ blood was drawn into BD P100 tubes (BD Diagnostics, Franklin Lake, NY, USA), which are specially designed for proteomics experiments, in that the EDTA (ethylenediaminetetraacetic acid) and protease inhibitor present in the tube prevent coagulation and stabilize the plasma proteome. Following phlebotomy the samples were centrifuged at $3,000 \times \mathrm{g}$ for 30 minutes at $10^{\circ} \mathrm{C}$, whereby the plasma was separated from the cellular fraction by aid of a mechanical separator, $100 \mu \mathrm{L}$ aliquots were stored at $-80^{\circ} \mathrm{C}$ until further use. For an overview of the workflow used in this analysis refer to Figure 1.

\subsection{Immuno-Depletion of High-Abundance Plasma Proteins.} Highly abundant plasma proteins were depleted using ProteoMiner protein enrichment kit (Bio-Rad Laboratories, Inc.), as per the manufacturer's instructions. $1 \mathrm{~mL}$ of plasma was used for the depletion and after the whole procedure, $300 \mu \mathrm{L}$ was eluted in elution reagent. After depletion protein concentration was measured using RC DC Protein assay kit (Bio-Rad Laboratories, Inc.).

2.4. Tryptic Digestion and iTRAQ Reagent Labelling. Equal amounts $(100 \mu \mathrm{g})$ of depleted plasma protein from six 
TABLE 1: Characteristic of study population.

(a) Criteria used for pregnancy with or without preeclampsia for the longitudinal study

\begin{tabular}{lcc}
\hline & Control & PE \\
\hline Maternal age (years) & $35.1 \pm 1.3$ & $35.1 \pm 6.8$ \\
Gestational age (weeks) & $38.8 \pm 0.8$ & $34.9 \pm 2.4$ \\
Systolic BP (mmHg) & $122.5 \pm 14.2$ & $188.8 \pm 18.6$ \\
Diastolic BP (mmHg) & $73.6 \pm 7.0$ & $111.8 \pm 14.1$ \\
Proteinuria (dipstick) & Negative & positive \\
\hline
\end{tabular}

(b) Gestational window selected for the current study $(n=6)$ who subsequently developed the preeclampsia

\begin{tabular}{lcc}
\hline & Control & PE \\
\hline Gestational age (years) & $12.4 \pm 1.2$ & $12.3 \pm 1.1$ \\
\hline
\end{tabular}

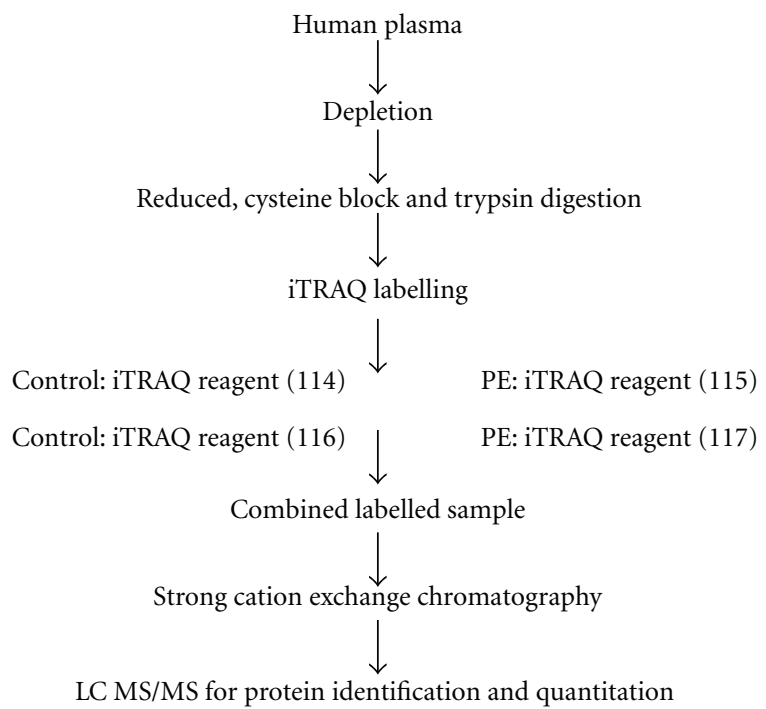

FIGURE 1: Workflow for quantitative proteomics using iTRAQ reagent. Equal amounts of plasma protein $(100 \mu \mathrm{g})$ from control and PE $(n=6)$ were pooled separately and duplicated (technical replicates), controls were labeled with 114 and 116 iTRAQ label, and PE were labeled with 115 and 117 iTRAQ label. The labeled samples were pooled and were subjected to a strong cation exchange chromatography to remove the excess label. Afterwards, LC-MALDI MS/MS was performed for protein identification and quantification.

of the PE cases and controls were pooled separately and splitted into two technical replicate for the iTRAQ labelling. These samples were denatured with $2 \%$ SPE in $500 \mathrm{mM}$ triethylammonium bicarbonate (TEAB) (Sigma-Aldrich) for 15 minutes at room temperature, following which they were reduced with $2 \mu \mathrm{L}$ of $50 \mathrm{mM}$ tris-(2-carboxyethyl) phosphine (TCEP) (Sigma-Aldrich) at $60^{\circ} \mathrm{C}$ for $1 \mathrm{~h}$ and were then alkylated with $10 \mathrm{mM} \mathrm{s}$-methyl methanethiosulfonate (MMTS) for 10 minutes in room temperature. After alkylation, the proteins were digested overnight at $37^{\circ} \mathrm{C}$ with $1 \mathrm{U} / \mu \mathrm{L}$ trypsin (TPCK treated) (Applied Biosystems, Foster City, CA 94404, USA). Peptides were labelled with one unit of iTRAQ Reagent Multiplex kit (Applied Biosystems, Foster City, CA 94404,
USA) that was reconstituted in $70 \mu \mathrm{L}$ of ethanol. iTRAQ labels 114 and 116 were used separately for labelling the pooled duplicated control sample (technical replicates), and 115 and 117 were used separately to label the pooled duplicate preeclampsia samples (technical replicates). The iTRAQ labelling reagent solution was added to the digest and incubated for $1 \mathrm{~h}$ at room temperature. To assess the accuracy of the ratiometric quantitation of iTRAQ reagent a split in signal was performed, and the data was corrected as described in detail by Unwin and colleagues [17].

2.5. Strong Cation Exchange Chromatography (SCX). Strong cation exchange chromatography was preformed to remove the excess iTRAQ reagent and interfering substances for the mass analysis. Dried peptides were resuspended in $200 \mathrm{~mL}$ of Buffer A and were loaded on polysulfoethyl A column (200 mm length $\times 4.6 \mathrm{id}, 5 \mu \mathrm{m}$ particle size, and $200 \AA \AA$ pore size) on a BioLC HPLC unit (Dionex). Buffer A consisted of $10 \mathrm{mM} \mathrm{KHPO}_{4}, 25 \%$ acetonitrile, and $500 \mathrm{mM} \mathrm{KCl}, \mathrm{pH} 3.0$, and Buffer B consisted of $10 \mathrm{mM} \mathrm{KH}_{2} \mathrm{PO}_{4}, 25 \%$ acetonitrile, and $500 \mathrm{mM} \mathrm{KCl}, \mathrm{pH}$ 3.0. The $60 \mathrm{~min}$ gradient comprised $100 \%$ Buffer A for $5 \mathrm{~min}, 5-30 \%$ Buffer B for $40 \mathrm{~min}, 30$ $100 \%$ Buffer B for $5 \mathrm{~min}, 100 \%$ Buffer B for $5 \mathrm{~min}$, and finally $100 \%$ Buffer A for $5 \mathrm{~min}$. Thirteen fractions were collected using a Foxy Jr. Fraction Collector (Dionex). Subsequently, 2 and 3 fractions were pooled according to the chromatogram profile based on the peak intensity, and all the products were dried in a vacuum concentrator, after which they were stored at $-20^{\circ} \mathrm{C}$ prior to mass spectrometric analysis.

2.6. Nano-LC-MALDI. Should we changeThe dried SCX iTRAQ-labeled peptides were dissolved in Buffer A which consists of $95 \% \mathrm{H}_{2} \mathrm{O}, 5 \%$ acetonitrile, $0.1 \%$ TFA and were loaded on C18 trap column $(1 \mathrm{~mm} \times 300 \mu \mathrm{m}$ i.d. column $)$ at $30 \mu \mathrm{l} / \mathrm{minand}$ separated on an analytical column $(150 \mathrm{~mm}$ $\times 100 \mu \mathrm{m}$ i.d. column) at $500 \mathrm{~nL} / \mathrm{min}$ using the LC Packings Ultimate system. The peptides were separated using a linearly increasing concentration of acetonitrile in Buffer B from $5 \%$ to $30 \%$ in $120 \mathrm{~min}$, and from $30 \%$ to $60 \%$ in $40 \mathrm{~min}$. The elute was mixed with matrix $(2 \mathrm{mg} / \mathrm{mL}$ alpha-cyano4-hydroxycinnamic acid in $80 \%$ acetonitrile, $0.1 \%$ TFA) at a flow rate of $800 \mathrm{nl} / \mathrm{min}$ and deposited on an OptiTOF LC/MALDI (Applied Biosystems) plate in 10s fractions, using an automatic robot, (Probot, Dionex).

2.7. MS and MS/MS. The mass spectrometer 4800 Plus MALDI TOF/TOF Analyzer (Applied Biosystems) was set to perform data acquisition in positive ion mode. An MS condition of 1000 shots per spectrum was used. Monoisotopic precursor selection for MS/MS was done by automatic precursor selection using an interpretation method using the 12 most intense peaks per spot with an MS/MS mode condition of 4000 laser shots per spectrum. Minimum peak width was one fraction, and mass tolerance was $80 \mathrm{ppm}$. Adduct tolerance $(\mathrm{m} / \mathrm{z}) \pm 0.003$. MSMS was done with a gas pressure of $2 \times 10^{-2}$ bar in the collision cell. Air was used as collision gas. 
2.7.1. Protein Identification and Database Searches. Protein identification and quantification were done by using the ProteinPilot software v2.0 (Applied Biosystems; MPE-Sciex). The search was performed against the human database of UniProtKB/Swiss-Prot (version 3.52, November 2008) from the EBI website (http://www.ebi.ac.uk./IPI/IPIhelp.html), and concatenated target-decoy database-search strategy was used to check the false positive rate [18].

2.8. Relative Quantitation Criteria. The Paragon algorithm $[19,20]$ in ProteinPilot v2.0 software was used as the default search program with digestion enzyme set as trypsin and methyl methanethiosulfonate as cysteine modification. The search also included the possibility of more than eighty biological modifications and amino acid substitution of up to two substitutions per peptide using the BLOSUM62 matrix. Data was normalized for loading error by bias correction calculated with ProGroup algorithm, and identified proteins with at least $95 \%$ confidence and with a ProtScore of 1.3 were reported. The results obtained from ProteinPilot v2.0 software were exported to Microsoft Excel for further analysis. The study was performed in double duplex manner, where PE samples were labelled with 115 and 117; control was labelled with 114 and 116. Peptides were selected based on the criteria defined in the Protein Pilot software, which means that all the peptides were included for quantitation with an exception for those without an iTRAQ modification or reporter ion; an area count less than 40 and peptides with $P$ value less than 0.001 were excluded [21]. As described by Gan and colleague in their study on estimation of relative quantitative ratio from iTRAQ experiments, we also used only peptides above or equal to $70 \%$ confidence level for the estimation of relative quantitation [22].

2.9. PANTHER Analysis. The PANTHER database was used to elucidate the molecular function, biological process, and signalling pathway associated with each individual protein (http://panther.appliedbiosystems.com/). (See Supplementary Material available online at doi:10.1155/2011/305964.)

\section{Results}

Samples were obtained from 6 cases which subsequently developed preeclampsia and 6 cases which delivered normal healthy babies. Care was taken to match both maternal and gestational age, to rule out any confounding influence of these two parameters.

Low-abundant-plasma proteins were enriched by using ProteoMiner protein enrichment kit. This was accomplished through the use of a large highly diverse bead-based library of combinatorial peptide ligands. When plasma was applied to the beads, a small fraction of the high-abundance proteins saturated their high-affinity ligands, and the excess high-abundance proteins were washed away. In addition a very small amount of high-abundance proteins and lowabundance proteins were concentrated on their specific affinity ligands. This provides for a significant enrichment of medium- and low-abundant plasma proteins.
The samples were pooled separately and duplicated in order to have more precise analytical replicate measurements. The iTRAQ analysis was done in double duplex style, the PE samples were labelled with iTRAQ 115 and 117 and the control samples with iTRAQ 114 and 116, using the workflow illustrated in Figure 1.

Following tandem MSMS, and by focussing on iTRAQ reporter ions in low molecular mass range $(114-117 \mathrm{Da})$ for quantification, we identified 64 proteins with $\geq 95 \%$ confidence. However, after manually rechecking the MS/MS data thoroughly peak by peak, only 57 out of 64 proteins $(92 \%)$ had a relative quantitation derived from the analysis of two or more peptides, while for 5 proteins, the quantitation was based on single peptide. For 2 proteins no quantitation could be ascertained by analysis using ProteinPilot. Figure 2 shows the MSMS spectrum of the precursor $([\mathrm{M}+\mathrm{H}]+, \mathrm{m} / \mathrm{z}$ 1259.67 Da). In low-mass region the reporter ions are seen, while the area under the curve was used for quantification. A concatenated target-decoy database search strategy was used to check the false positive rate in our case, it was found to be $0 \%$, which boosted the reliability of our data.

As we did the experiment in double duplex manner PE (115 and 117) and control (114 and 116), it was possible to estimate the cutoff point for the differentially expressed protein in our sample. Based on 64 relatively abundant protein ratios from $\mathrm{PE}$ and control an average variation of $4.4 \%$ was $( \pm 0.04)$ measured. If the cutoff was set at $10 \%$ average variance then only $74 \%$ of the proteins would fall within this variation range, but when it was increased to $20 \%$ then about $98 \%$ of the proteins fall in this variation range.

So the cutoff point in this experiment was set at $20 \%$ $( \pm 0.2)$. That means that any relative change in protein ratio below or above \pm 1.2 -fold was considered as differentially under or overexpressed Figure 3.

The functional distribution of these proteins is illustrated in Figure 4. For this interpretation, an analysis of 64 proteins was performed using the PANTHER classification system, which sorts the proteins into respective classes based on their biological process.

It is of interest that three of the major groups involve cell adhesion molecules (13\%), extracellular matrix proteins (13\%), and member of the protease family (13\%), and factors that are known to be aberrant in placental insufficiency apparent in preeclampsia. Furthermore, large groups were found to involve signalling molecules (13\%) and carrier proteins (20\%).

A list of 10 of the upregulated proteins with fold change more than 1.2 is provided in Table 2. A PANTHER subanalysis for pathway association of these 10 proteins indicates that $33 \%$ are associated with blood coagulation, $33 \%$ with plasminogen activation, $17 \%$ with angiotensin II, and $17 \%$ with integrin function shown in Figure 5.

\section{Discussion}

The comparative quantitative assessment of serum or plasma proteins via iTRAQ isobaric labelling combined with MALDI-TOF/TOF analysis has suggested to be suitable for 


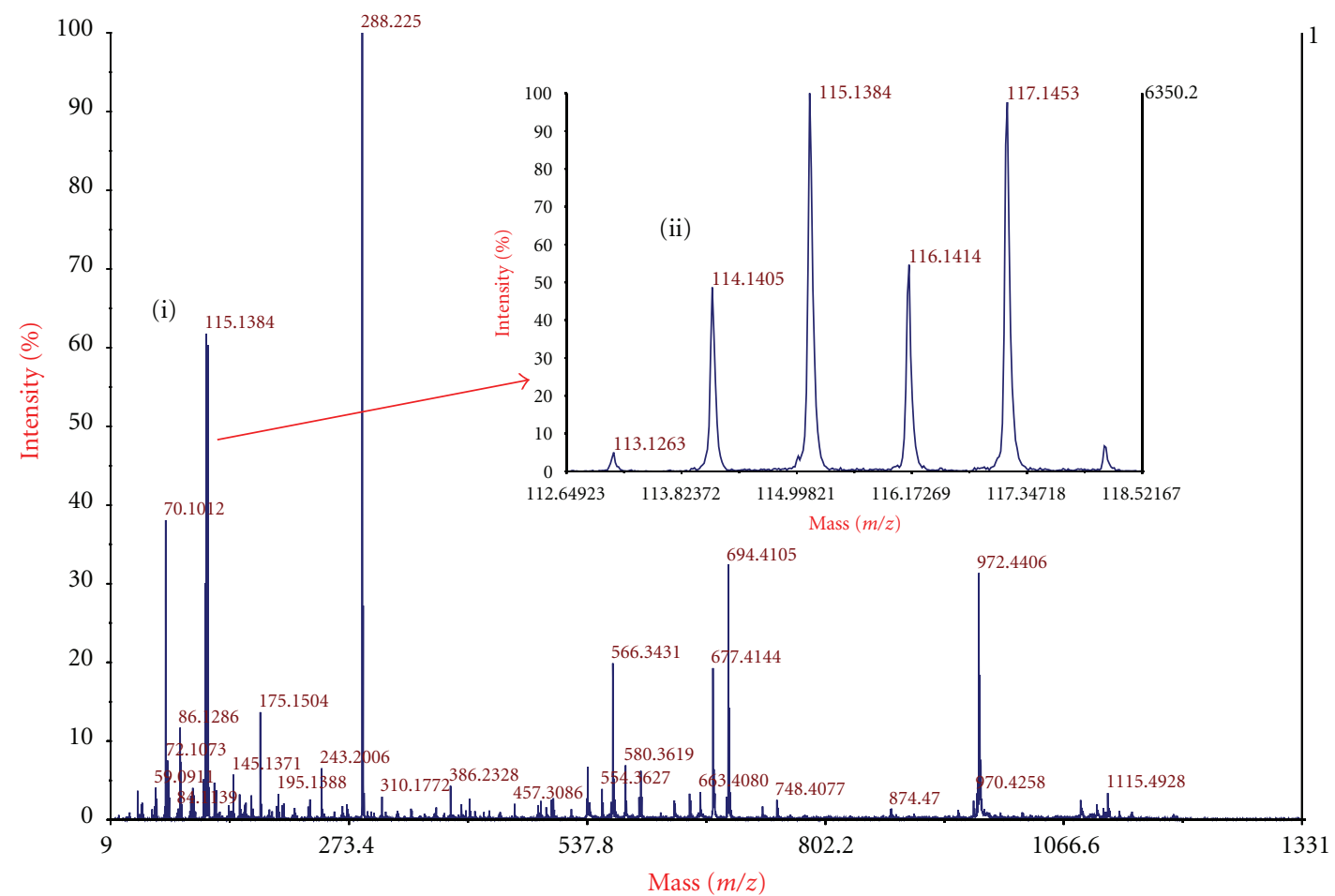

FIGURE 2: Components of the spectrum illustrated are (i) MS and MS spectrum of the precursor ([M + H]+, m/z 1259.67 Da) (ii) low-mass region showing the reporter ions used for quantitation. The peptide is labeled by isobaric tags at both the N-terminus and C-terminal lysine side chain. The precursor ion and all the fragment ions therefore contain all four members of the tag set, but remain isobaric. The MS and MS spectrum was obtained from the singly charged $[\mathrm{M}+\mathrm{H}]+$ peptide using a 4800 MALDI TOF-TOF Analyzer.

TABLE 2: List of protein up regulated in ITRAQ experiment with relative protein ratio ( $P$ value 0.001$)$.

\begin{tabular}{lcccc}
\hline$N$ & Acc. no. & Protein names & Biological processes & Molecular function \\
\hline 1 & P02679 & Fibrinogen Fragment D & Signal transduction & Calcium ion binding \\
2 & P10909 & Clusterin isoform 2 & Compliment activation & Misfolded protein binding \\
3 & P02647 & Apolipoprotein A-I & Signal transduction & Receptor binding \\
4 & P02751 & Fibronectin & Angiogenesis & Collagen binding \\
5 & P01019 & Angiotensinogen & Blood vessel remodeling & Receptor binding \\
6 & P09382 & Galectin 3 binding & Apoptosis & Galactoside binding \\
7 & P00750 & Plasminogen & Proteolysis & Protein binding \\
8 & P02787 & Transferrin & Iron ion transporter & Ferric ion binding \\
9 & P04003 & C4 beta binding & Compliment activation & Protein binding \\
\hline
\end{tabular}

the detection of biomarkers. This is largely due to the highly reproducible nature of the system, which exhibits little runto-run variation, determined after a lengthy comparison of pooled and individual samples of case and control, yielding a coefficient of variation of $11.7 \%$ [15].

In a previous proof-of-principle study, we examined whether this approach was suitable for the detection of screening markers that could be used to screen for pregnancies with a Down syndrome fetus [16]. This analysis indicated that this approach may indeed be valid, as we found a known screening marker, namely, $\beta$ hCG ( $\beta$ human chorionic gonadotropin), amongst the pool of elevated proteins we detected.

In our current study, we have now assessed whether this method could be suitable for the detection of biomarkers useful for PE screening. In our analysis we identified over 10 proteins whose concentration was altered in the plasma of pregnancies that subsequently developed $\mathrm{PE}$ when compared to those with normal deliveries. Amongst this group, we observed previously described glycoproteins [12] reported to be involved in the pathogenesis of PE such as fibrinogen, galectin, transferrin, hemopexin, and clusterine. Fibrinogen 


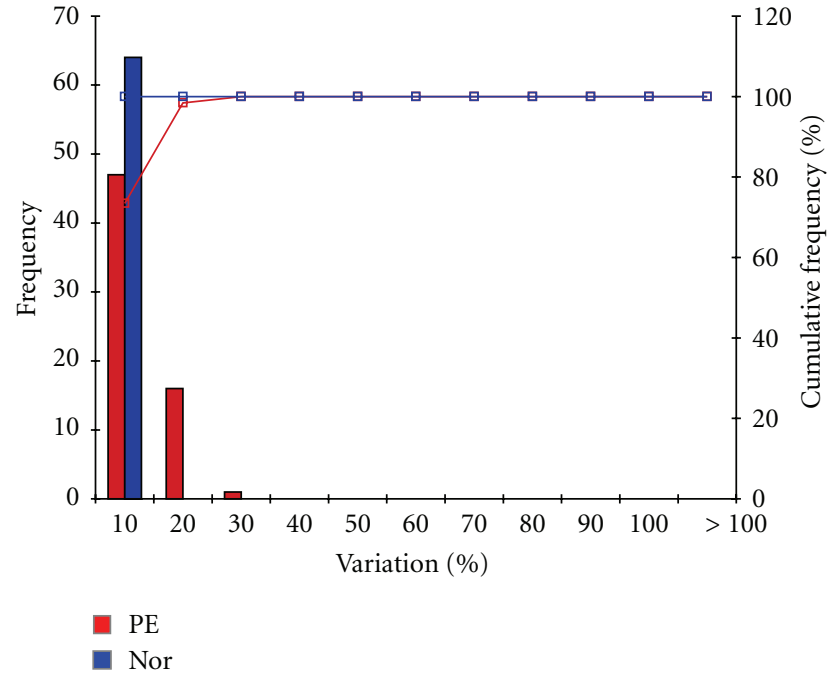

FIgURe 3: Frequency distribution (bars) from both PE and control replicates across different ranges of variation. The cumulative percentage (lines) is defined as the cumulative number of proteins falling within the defined variation range against the total number of proteins.

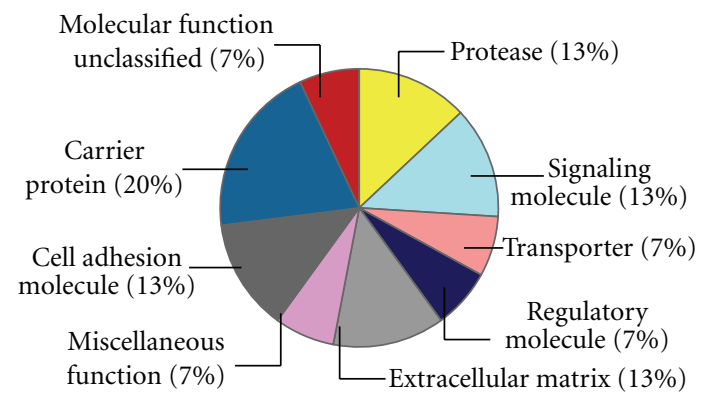

FIgURE 4: Number of plasma protein identified using iTRAQ reagent. In total, 64 proteins were identified. Shown above is the classification of these proteins in different categories based on molecular function.

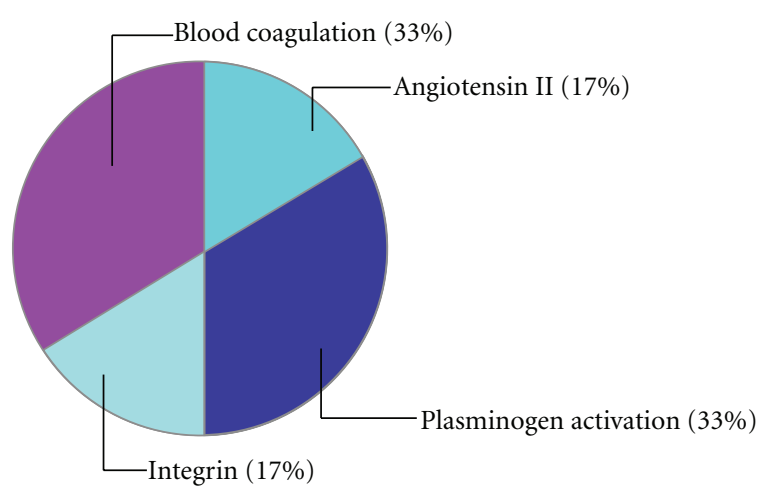

Figure 5: PANTHER analysis for pathway. In total, 10 proteins were identified as elevated. Shown above is the different signaling pathways hits by these protein. is present in the extracellular matrix of the placenta and plays an important role in cellular and matrix interaction. It can also play a role in inflammatory response, as an increase in level of fibrinogen can lead to adhesion of leukocytes and platelets to the endothelium [23]. Pathway analysis has shown that $33 \%$ of proteins are associated with blood coagulation pathway [24]. This suggests a larger-thanexpected role that fibrinogen is playing in the pathogenesis of $\mathrm{PE}$ and needs to be further investigated in detail.

Extracellular matrix protein fibronectin was also found to be overexpressed. Fibronectin is an important glycoprotein with a major role in early embryogenesis, trophoblast invasion, and vascular remodelling [25, 26]. Fibronectin has been suggested to be a potential predictive marker for PE. It is also of interest that $13 \%$ of proteins we detected are associated with integrin pathway signalling, of which fibronectin is an important component.

We also found elevation in the level of apolipoprotein A1 which is involved in lipid metabolism. We also found altered level ins protease inhibitor (serpin) family member angiotensinogen and proteolytic factor like plasmin. Complement factor C4 beta, which plays important role in compliment activation was also observed to be elevated in our study.

In a recent study Blumenstein and colleagues [12] have also observed an elevation in clusterin prior to onset of preeclampsia, thereby suggesting that it may also serve as a predictive marker. In our study we were, however, able to detect this elevation at a much earlier time-point in gestation in those cases that subsequently developed PE.

It is of interest that the blood pressure regulating molecule angiotensinogen was also amongst the group of proteins we observed to be increased. This important molecule has previously reported to be elevated in manifest PE. The presence of this important screening marker in our pool of elevated plasma proteins suggests that the strategy we have chosen for the identification of new biomarkers is functional and worthy of further pursuit [27].

That our assay is indeed capable of detecting proteins of placental origin is illustrated by presence of galectin, a placentally derived glycoprotein. This molecule is important in pregnancy by playing a potential role in preventing the attack from maternal immune system on the developing fetus, which can be seen as foreign semiallograft [28].

That the conditions leading to $\mathrm{PE}$ are multifactorial is suggested by our observation that it may also involve factors such as apolipoprotein A-I, whose altered production might impair cholesterol transport, thereby contributing to arterial damage in those cases which develop PE [29].

The underlying role of inflammatory processes are is also underscored by our observed elevation in complement $\mathrm{C} 4$ beta. Although this elevation may be largely contributed by the mother, it may be a reflection upon a maternal response to the increased release of placental debris which has been suggested to occur in pregnancies with PE.

Our study supports the current view that the iTRAQ approach is very useful for the detection of biomarkers, largely due to its reproducibility and robustness. However, in order to assess the clinical utility of these new biomarkers, 
we will need to further investigate them using modern technologies such as selected reaction monitoring (SRM), which has emerged as the method of choice for biomarkers validation using mass spectroscopy [30,31].

As very few specific biomarkers currently exist to assist with the screening of pregnancy-related disorders such preeclampsia or preterm labour, it is possible that the approach we have described in this study will be useful for the discovery of such important clinical tools.

\section{Acknowledgments}

The authors thank Mrs. Vivian Kiefer for her technical assistance and Professor E. Palmer and Dr. D. Huang for the proof reading of the paper. This study was supported by PREGENESYS (ref. no. 37244), Sixth Frame Work (FP6) grant.

\section{References}

[1] M. C. Hogan, K. J. Foreman, M. Naghavi et al., "Maternal mortality for 181 countries, 1980-2008: a systematic analysis of progress towards Millennium Development Goal 5," The Lancet, vol. 375, no. 9726, pp. 1609-1623, 2010.

[2] B. Jim, S. Sharma, T. Kebede, and A. Acharya, "Hypertension in pregnancy: a comprehensive update," Cardiology in Review, vol. 18, no. 4, pp. 178-189, 2010.

[3] E. A. Steegers, "Pre-eclampsia," The Lancet, vol. 376, no. 9741, pp. 631-644, 2010.

[4] M. Silasi, B. Cohen, S. A. Karumanchi, and S. Rana, "Abnormal placentation, angiogenic factors, and the pathogenesis of preeclampsia," Obstetrics and Gynecology Clinics of North America, vol. 37, no. 2, pp. 239-253, 2010.

[5] S. Y. Kim, H. M. Ryu, J. H. Yang et al., "Maternal serum and amniotic fluid inhibin A levels in women who subsequently develop severe preeclampsia," Journal of Korean Medical Science, vol. 21, no. 3, pp. 452-456, 2006.

[6] S. Y. Kim, H. M. Ryu, J. H. Yang et al., "Increased sFlt-1 to PlGF ratio in women who subsequently develop preeclampsia," Journal of Korean Medical Science, vol. 22, no. 5, pp. 873-877, 2007.

[7] K. Spencer, N. J. Cowans, and A. Stamatopoulou, "ADAM12s in maternal serum as a potential marker of pre-eclampsia," Prenatal Diagnosis, vol. 28, no. 3, pp. 212-216, 2008.

[8] R. Akolekar, A. Syngelaki, J. Beta, R. Kocylowski, and K. H. Nicolaides, "Maternal serum placental protein 13 at 11-13 weeks of gestation in preeclampsia," Prenatal Diagnosis, vol. 29, no. 12, pp. 1103-1108, 2009.

[9] A. Khalil, N. J. Cowans, K. Spencer, S. Goichman, H. Meiri, and K. Harrington, "First trimester maternal serum placental protein 13 for the prediction of pre-eclampsia in women with a priori high risk," Prenatal Diagnosis, vol. 29, no. 8, pp. 781789, 2009.

[10] K. Spencer, N. J. Cowans, and K. H. Nicolaides, "Low levels of maternal serum PAPP-A in the first trimester and the risk of pre-eclampsia," Prenatal Diagnosis, vol. 28, no. 1, pp. 7-10, 2008.

[11] E. R. Norwitz, "Defective implantation and placentation: laying the blueprint for pregnancy complications," Reproductive BioMedicine Online, vol. 13, no. 4, pp. 591-599, 2006.
[12] M. Blumenstein, M. T. McMaster, M. A. Black et al., "A proteomic approach identifies early pregnancy biomarkers for preeclampsia: novel linkages between a predisposition to preeclampsia and cardiovascular disease," Proteomics, vol. 9, no. 11, pp. 2929-2945, 2009.

[13] P. L. Ross, Y. N. Huang, J. N. Marchese et al., "Multiplexed protein quantitation in Saccharomyces cerevisiae using aminereactive isobaric tagging reagents," Molecular and Cellular Proteomics, vol. 3, no. 12, pp. 1154-1169, 2004.

[14] P. K. Chong, C. S. Gan, T. K. Pham, and P. C. Wright, "Isobaric tags for relative and absolute quantitation (iTRAQ) reproducibility: implication of multiple injections," Journal of Proteome Research, vol. 5, no. 5, pp. 1232-1240, 2006.

[15] X. Song, J. Bandow, J. Sherman et al., "iTRAQ experimental design for plasma biomarker discovery," Journal of Proteome Research, vol. 7, no. 7, pp. 2952-2958, 2008.

[16] V. Kolla, P. Jenö, S. Moes et al., "Quantitative proteomics analysis of maternal plasma in Down syndrome pregnancies using isobaric tagging reagent (iTRAQ)," Journal of Biomedicine \& Biotechnology, vol. 2010, Article ID 952047, 10 pages, 2010.

[17] R. D. Unwin, A. Pierce, R. B. Watson, D. W. Sternberg, and A. D. Whetton, "Quantitative proteomic analysis using isobaric protein tags enables rapid comparison of changes in transcript and protein levels in transformed cells," Molecular and Cellular Proteomics, vol. 4, no. 7, pp. 924-935, 2005.

[18] J. E. Elias and S. P. Gygi, “Target-decoy search strategy for increased confidence in large-scale protein identifications by mass spectrometry," Nature Methods, vol. 4, no. 3, pp. 207214, 2007.

[19] I. V. Shilov, S. L. Seymourt, A. A. Patel et al., "The paragon algorithm, a next generation search engine that uses sequence temperature values sequence temperature values and feature probabilities to identify peptides from tandem mass spectra," Molecular and Cellular Proteomics, vol. 6, no. 9, pp. 1638-1655, 2007.

[20] H. Mi, N. Guo, A. Kejariwal, and P. D. Thomas, "PANTHER version 6: protein sequence and function evolution data with expanded representation of biological pathways," Nucleic Acids Research, vol. 35, no. 1, pp. D247-D252, 2007.

[21] A. Glen, C. S. Gan, F. C. Hamdy et al., "ITRAQ-facilitated proteomic analysis of human prostate cancer cells identifies proteins associated with progression," Journal of Proteome Research, vol. 7, no. 3, pp. 897-907, 2008.

[22] C. S. Gan, K. C. Poh, K. P. Trong, and P. C. Wright, "Technical, experimental, and biological variations in isobaric tags for relative and absolute quantitation (iTRAQ)," Journal of Proteome Research, vol. 6, no. 2, pp. 821-827, 2007.

[23] M. W. Mosesson, "Fibrinogen and fibrin structure and functions," Journal of Thrombosis and Haemostasis, vol. 3, no. 8, pp. 1894-1904, 2005.

[24] N. E. Kirschbaum, M. W. Mosesson, and D. L. Amrani, "Characterization of the $\gamma$ chain platelet binding site on fibrinogen fragment D," Blood, vol. 79, no. 10, pp. 2643-2648, 1992.

[25] C. Benoit, J. Zavecz, and Y. Wang, "Vasoreactivity of Chorionic Plate Arteries in Response to Vasoconstrictors Produced by Preeclamptic Placentas," Placenta, vol. 28, no. 5-6, pp. 498504, 2007.

[26] M. M. G. Leeflang, J. S. Cnossen, J. A. M. van der Post, B. W. J. Mol, K. S. Khan, and G. ter Riet, "Accuracy of fibronectin tests for the prediction of pre-eclampsia: a systematic review," European Journal of Obstetrics Gynecology and Reproductive Biology, vol. 133, no. 1, pp. 12-19, 2007. 
[27] L. Anton, D. C. Merrill, L. A. A. Neves et al., "Activation of local chorionic villi angiotensin II levels but not angiotensin (1-7) in preeclampsia," Hypertension, vol. 51, no. 4, pp. 10661072, 2008.

[28] E. L. Skornicka, N. Kiyatkina, M. C. Weber, M. L. Tykocinski, and P. H. Koo, "Pregnancy zone protein is a carrier and modulator of placental protein-14 in T-cell growth and cytokine production," Cellular Immunology, vol. 232, no. 1-2, pp. 144-156, 2004.

[29] K. R. Atkinson, M. Blumenstein, M. A. Black et al., "An altered pattern of circulating apolipoprotein E3 isoforms is implicated in preeclampsia," Journal of Lipid Research, vol. 50, no. 1, pp. 71-80, 2009.

[30] V. Lange, P. Picotti, B. Domon, and R. Aebersold, "Selected reaction monitoring for quantitative proteomics: a tutorial," Molecular Systems Biology, vol. 4, article no. 222, 2008.

[31] P. Picotti, B. Bodenmiller, L. N. Mueller, B. Domon, and R. Aebersold, "Full dynamic range proteome analysis of S. cerevisiae by targeted proteomics," Cell, vol. 138, no. 4, pp. 795806, 2009. 


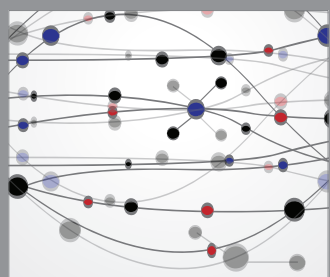

The Scientific World Journal
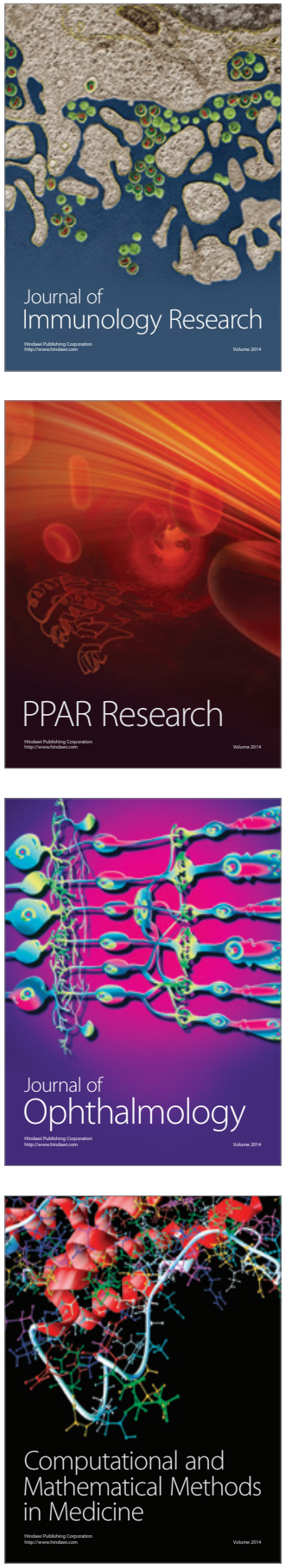

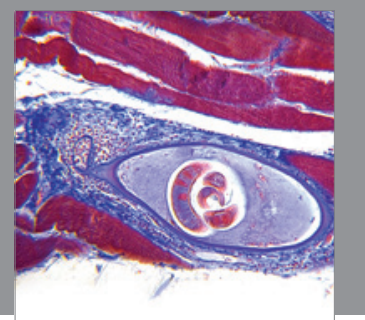

Gastroenterology

Research and Practice
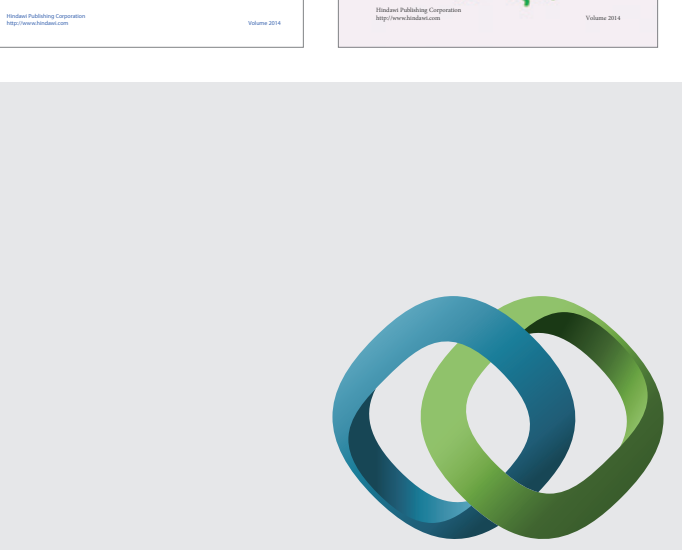

\section{Hindawi}

Submit your manuscripts at

http://www.hindawi.com
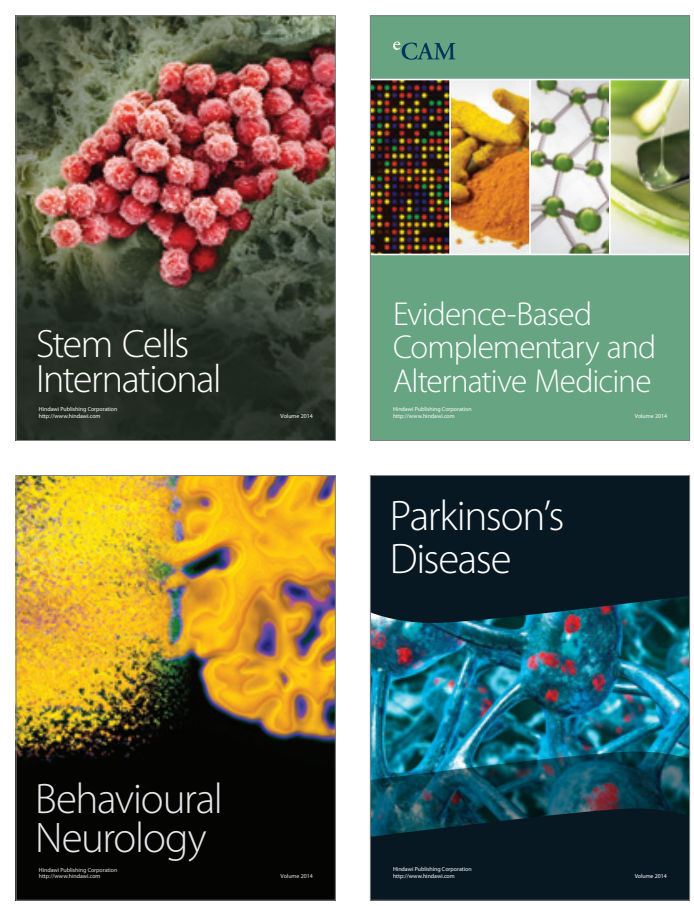

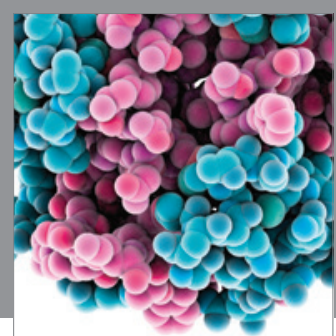

Journal of
Diabetes Research

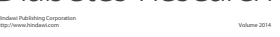

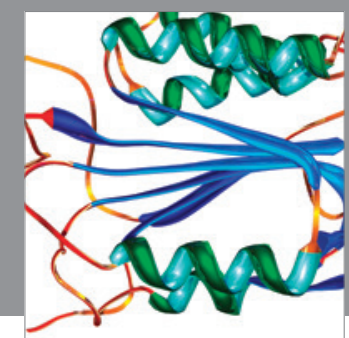

Disease Markers
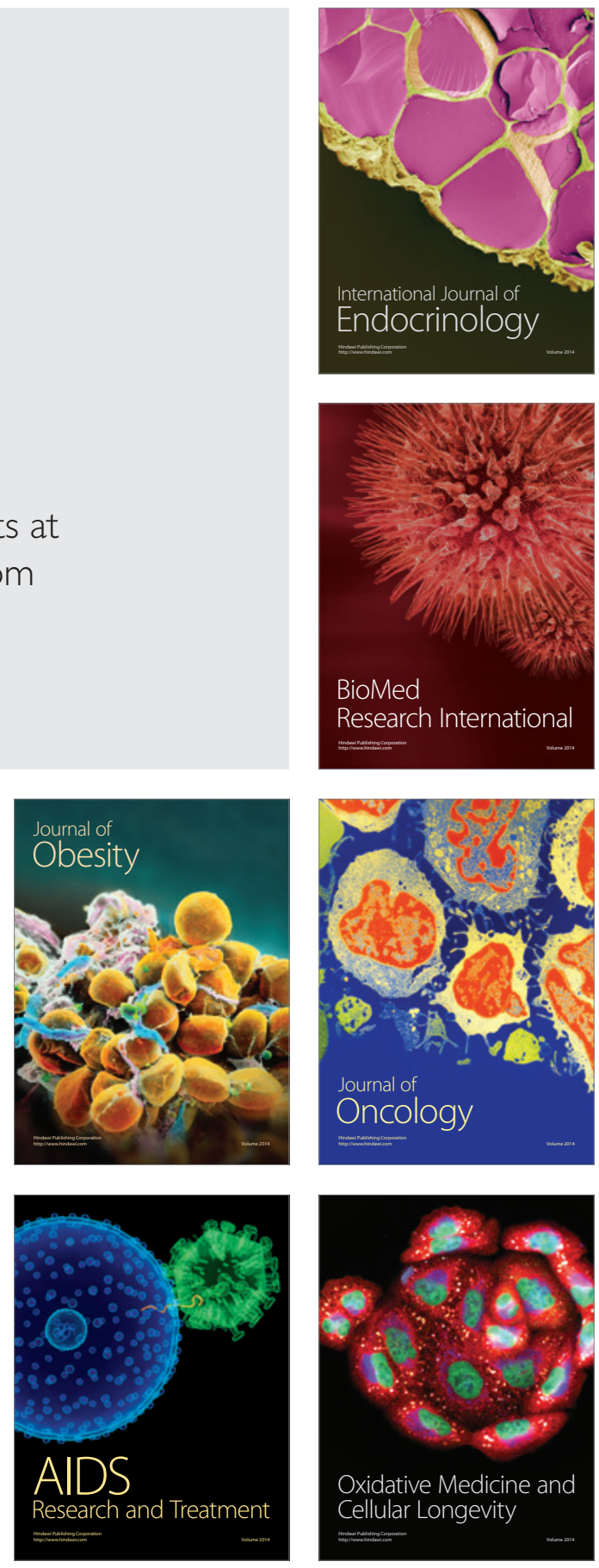